\title{
EFICÁCIA AGRONÔMICA DE FORMULAÇÕES E DOSES DE TRIFLURALIN NA CULTURA DA SOJA
}

\author{
Julio P. Laca-Buendia ${ }^{1}$ e Joel C. Ferreira ${ }^{2}$
}

\author{
${ }^{\prime}$ Eng $^{\circ}$. Agro ${ }^{\circ}$., M. Sc., Pesquisador. EPAMIG/DPPE. Av. José Cândido da Silveira, 1647 (Cidade Nova) \\ Belo Horizonte, MG 30170-000. ilaca.geo@yahoo.com \\ ${ }^{2}$ Eng $^{\circ}$. Agro ${ }^{\circ}$., Pesquisador. EPAMIG/CTTP/FEGV. Caixa Postal 351. Uberaba, MG 38001-970
}

\begin{abstract}
RESUMO
O objetivo do presente trabalho foi avaliar a eficácia das diferentes formulações de trifluralin para o controle de poáceas na cultura da soja (cv. Garimpo), em Latossolo Vermelho-Amarelo, textura arenosa, em Uberaba, MG. Os tratamentos testados foram: trifluralin GrDA (500 g/l) a 750 e $1000 \mathrm{~g} / \mathrm{ha}$, trifluralin CE $(600 \mathrm{~g} / \mathrm{l})$ a $720 \mathrm{~g} / \mathrm{ha}$ e trifluralin CE (445 g/ 1) a $667 \mathrm{~g} / \mathrm{ha}$, testemunhas capinada e sem capina. O ensaio foi instalado no desenho experimental de blocos casualizados, com quatro repetições. Observou-se que as duas formulações do herbicida trifluralin (GrDA e CE), nas doses utilizadas, foram eficientes (80-90\%) para o controle de Brachiaria decumbens e B. plantaginea. As características agronômicas da soja, como produtividade, altura das plantas, inserção da primeira vagem, peso de sementes e índice de colheita mecânica não foram afetados de forma distinta pelas formulações e doses usadas. Os herbicidas usados também não provocaram toxicidade à soja. Entretanto, observou-se uma leve superioridade da formulação CE no controle inicial de $B$. decumbens (15 dias após aplicação) e final de B. plantaginea (45 dias após aplicação).
\end{abstract}

Palavras-chave: Glycine max, Brachiaria decumbens, Brachiaria plantaginea.

\section{ABSTRACT \\ Efficacy of trifluralin formulations and doses in soybean}

The objective of this study was to evaluate the efficacy of different formulations of trifluralin to control grass weeds in soybean (cv. Garimpo) on a Red-Yellow Latossolo, with a sandy texture, in Uberaba, MG, Brazil. The treatments were: trifluran WG (500 g/l) at 750 and $1000 \mathrm{~g} / \mathrm{ha}$, trifluralin EC $(600 \mathrm{~g} / \mathrm{l})$ at $720 \mathrm{~g} / \mathrm{ha}$ and trifluralin EC (445 g/l) at $667 \mathrm{~g} / \mathrm{ha}$, as well as an unweeded and a weeded control. The experimental design was a complete randomized block with four replications. The two trifluralin formulations (WG and EC) controlled 80-90\% of Brachiaria decumbens and B. plantaginea. The agronomic characteristics of soybean such as productivity, plant height, first pod insertion, seed weight, and mechanical harvest indice, were not affected by the formulations nor doses of trifluralin. The herbicide also did not provoke toxicity to the soybean. However, a slight superiority of the EC formulation in the early control of Brachiaria decumbens (15 days after herbicide application) and late control (45 days after herbicide application) of $B$. plantaginea was noticed.

Key words: Glycine max, Brachiaria decumbens, Brachiaria plantaginea. 


\section{INTRODUÇÃO}

Nos últimos anos, em virtude da importância da soja na agricultura nacional e nos programas de apoio governamental, observou-se uma expansão dessa cultura no Estado de Minas Gerais, basicamente nas regiões do Triângulo Mineiro e Alto Paranaíba, onde se concentra cerca de $90 \%$ da produção do Estado (Sediyama \& Reis, 1990).

Estima-se que, no Triângulo Mineiro, a competição total de plantas daninhas com a cultura provoca $29 \%$ de perdas na produtividade e os maiores rendimentos são obtidos quando se mantem a cultura livre de plantas daninhas por 40 dias após a emergência (Maia et al., 1991).

Para o controle de plantas daninhas, na cultura de soja, vários pesquisadores têm estudado a aplicação de herbicidas em pré-plantio incorporado (Lorenzi \& Davis, 1976; Victória Filho et al., 1976; Maia,1978; Rafael et al., 1978; Melhorança \& Victória Filho, 1982; Silva \& Kishino, 1984; Scalea, 1986; Machado Neto et al., 1988; Rodrigues \& Coelho, 1988; Maia, et al., 1991 e Dario et al., 1993).

O objetivo do presente ensaio de campo foi avaliar a eficácia de diferentes formulações de trifluralin para o controle de poáceas, através da aplicação em pré-plantio incorporado na cultura de soja, em áreas de cerrado.

\section{MATERIAL E MÉTODOS}

O ensaio de campo foi instalado na Fazenda da Escola Agrotécnica Federal de Uberaba, MG., no ano agrícola de 1994/95, em solo de Cerrado do tipo Latossolo VermelhoAmarelo, textura arenosa, com $73 \%$ de areia, $17 \%$ de argila, $10 \%$ de limo, contendo $1,9 \%$ de matéria orgânica, $\mathrm{pH}$ (água) $=9,1$ e $69 \%$ de saturação de bases da CTC a pH=7,0.

Utilizou-se a cultivar Garimpo, semeada em 21/12/ 94 , no espaçamento de $0,5 \mathrm{~m}$ entre linhas, com semeadora de tração mecânica de quatro linhas, em uma densidade de 30 sementes por metro linear.

A adubação usada na semeadura foi de $400 \mathrm{~kg} / \mathrm{ha}$ da fórmula 0:20:20 de NPK.
O ensaio foi instalado no desenho experimental de blocos casualizados, com quatro repetições. As parcelas, de 2 $\mathrm{m} \times 7 \mathrm{~m}$, proporcionaram área total de $14 \mathrm{~m}^{2}$. Foram colhidas apenas as duas linhas centrais, eliminando-se $0,5 \mathrm{~m}$ em cada uma das extremidades, constituindo uma área útil de 1 $\mathrm{m} \times 6 \mathrm{~m}=6 \mathrm{~m}^{2}$ por parcela.

Os herbicidas (Tabela 1) foram aplicados em 16/12/ 94 na modalidade de pré-plantio incorporado. A incorporação foi feita até $10 \mathrm{~cm}$ de profundidade, utilizando-se uma grade de discos. O solo estava úmido e sob a temperatura de $39^{\circ} \mathrm{C}$ a $5 \mathrm{~cm}$ de profundidade. Foi utilizado um pulverizador costal pressurizado a $\mathrm{CO}_{2}$, dotado de barra com quatro bicos do tipo leque 80.04 a $50 \mathrm{~cm}$ do solo, aplicando volume de calda de $280 \mathrm{l} / \mathrm{ha}$, sob pressão de $2,8 \mathrm{kgf} / \mathrm{cm}^{2}$, entre $14: 15$ e 14:30 horas. No momento da aplicação o céu apresentava-se com céu parcialmente nublado, vento moderado, com temperatura do ar de $35^{\circ} \mathrm{C}$ e umidade relativa de $66 \%$. As precipitações ocorridas durante o ensaio estão apresentadas na Tabela 2 .

Aos 35 dias após a aplicação dos tratamentos foi aplicado o herbicida fomesafen $(250 \mathrm{~g} / \mathrm{l})$ na dose de $250 \mathrm{~g} / \mathrm{ha}$, em pós-emergência, em toda a área experimental, para o controle das plantas daninhas dicotiledôneas presentes.

As avaliações visuais de intoxicação das plantas de soja foram realizadas aos 15 e 30 dias após a aplicação, através da escala visual EWRC ( 1 a 9 ) onde 1 corresponde à nenhuma injúria e 9 corresponde à morte da planta.

Aos 30 e 45 dias após a aplicação realizaram-se avaliações da eficácia de controle das plantas daninhas poáceas, utilizando-se a escala percentual de 0 a $100 \%$, onde $0 \%$ corresponde a nenhum controle e $100 \%$ ao controle total para cada espécie. $\mathrm{Na}$ testemunha sem capina foi realizada a contagem de plantas por espécie, dentro da área útil de cada parcela, em $1 \mathrm{~m}^{2}$.

As espécies de plantas daninhas encontradas foram: Brachiaria decumbens (capim-braquiária), com 52 perfilhos/ $\mathrm{m}^{2}$ e Brachiaria plantaginea (capim-marmelada), com 21 perfilhos $/ \mathrm{m}^{2}$.

Antes da colheita foi realizada uma avaliação de praticidade para colheita mecânica, utilizando-se a escala

Tabela 1 - Tratamentos utilizados no experimento. Uberaba-MG,1994/95.

\begin{tabular}{|c|c|c|c|c|}
\hline \multirow[b]{2}{*}{ Tratamento } & \multirow[b]{2}{*}{$\begin{array}{c}\text { Concentração } \\
(\mathrm{g} / \mathrm{l})\end{array}$} & \multirow[b]{2}{*}{$\begin{array}{l}\text { Produto } \\
\text { comercial }\end{array}$} & \multicolumn{2}{|c|}{ Dose } \\
\hline & & & $\begin{array}{l}\text { Produto técnico } \\
\text { (g/ha) }\end{array}$ & $\begin{array}{c}\text { Produto comercial } \\
\text { (I/ha) }\end{array}$ \\
\hline $\begin{array}{l}\text { Trifluralin GrDA } \\
\end{array}$ & 500 & Gramat & 750 & 1,5 \\
\hline Trifluralin GrDA & 500 & Gramat & 1000 & 2,0 \\
\hline Trifluralin CE & 600 & Premerlin & 720 & 1,2 \\
\hline Trifluralin CE & 445 & Trifluralina Defensa & 667 & 1,5 \\
\hline Testemunha capinada & --- & --- & --- & --- \\
\hline Testemunha sem capina & --- & --- & --- & --- \\
\hline
\end{tabular}


Eficácia agronômica de formulações e doses...

Tabela 2. Precipitação, em mm, durante o decorrer do experimento no campo. Uberaba, MG. 1994/95

\begin{tabular}{lcccc}
\hline \multicolumn{1}{c}{ Mês } & $\mathbf{1}^{\mathbf{0}}$ Decêndio & $\mathbf{2}^{\mathbf{0}}$ Decêndio & $\mathbf{3}^{\mathbf{0}}$ Decêndio & Total \\
\hline Dezembro & 132,0 & 43,2 & 107,8 & 283,0 \\
Janeiro & 112,0 & 26,2 & 102,6 & 240,8 \\
Fevereiro & 185,2 & 81,8 & 31,4 & 298,4 \\
Março & 48,2 & 43,8 & 78,2 & 170,2 \\
Abril & 32,4 & 33,8 & 31,0 & 97,2 \\
\hline Total & & & 1089,6 \\
\hline
\end{tabular}

visual de 1 a 5 , em que: $1=$ impossível $(100 \%$ a $80 \%$ de infestação), $2=$ alta infestação (79\% a 60\%), $3=$ infestação razoável ( $59 \%$ a $40 \%), 4=$ boa colheita (39\% a 20\%) 5- excelente/ótima ( $19 \%$ a $0 \%$ de infestação).

Por ocasião da colheita (30/04/95) foi realizada a contagem do número de plantas nas duas fileiras centrais $\left(3 \mathrm{~m}^{2}\right)$ e a pesagem dos grãos da área útil de cada parcela, medição de altura das plantas e altura de inserção da primeira vagem. Coletaram-se vagens de dez plantas aleatoriamente, dentro de cada parcela, sendo escolhidas ao acaso 100 sementes para determinar seu peso.

\section{RESULTADOS E DISCUSSÃO}

As condições de campo reinantes durante a condução do ensaio foram favoráveis tanto para o desenvolvimento da cultura de soja, bem como para uma boa atividade dos herbicidas no controle de plantas daninhas.

Não houve diferença significativa em termos de pro- dutividade da soja entre os tratamentos com herbicidas e a testemunha capinada, porém todos apresentaram maior produtividade em relação à testemunha sem capina (Tabela 3). As poáceas presentes na área foram altamente competitivas com a cultura, pois apresentaram uma redução de $1415 \mathrm{~kg}$ / ha na testemunha sem capina (Tabela 3 ).

A altura das plantas, altura de inserção da primeira vagem e peso de 100 sementes não diferenciaram significativamente entre os tratamentos testados (Tabelas 3 e 4).

Os herbicidas propiciaram um melhor índice de colheita mecânica em relação à testemunha sem capina, porém esse índice nestes tratamentos foi inferior à testemunha capinada (Tabela 4). Nenhum herbicida causou toxicidade às plantas de soja (Tabela 4).

As formulações GRDA e CE de trifluralin, nas doses utilizadas, apresentaram um bom controle (80-90\%) de Brachiaria decumbens e B. plantaginea (Tabela 5). A formulação CE apresentou uma leve superioridade no controle inicial de Brachiaria decumbens e no controle inicial de $B$. plantaginea na cultura da soja, sem, entretanto, afetar o desempenho agronômico desta cultura.

Tabela 3. Efeito de formulações de trifluralin e doses nas características agronômicas da soja. Uberaba, MG. $1994 / 95$.

\begin{tabular}{|c|c|c|c|c|c|c|}
\hline \multirow{2}{*}{ Tratamento } & \multirow{2}{*}{$\begin{array}{l}\text { Dose } \\
\text { (g/ha) }\end{array}$} & \multicolumn{2}{|c|}{ Plantas de soja $/ \mathrm{m}^{2}$} & \multirow{2}{*}{$\begin{array}{l}\text { Produção } \\
\text { (kg/ha) }\end{array}$} & \multirow{2}{*}{$\begin{array}{l}\text { Altura das } \\
\text { plantas } \\
(\mathrm{cm})\end{array}$} & \multirow{2}{*}{$\begin{array}{l}\text { Altura Inserção } \\
\text { de } 1^{\mathrm{a}} \text { vagem }(\mathrm{cm})\end{array}$} \\
\hline & & Inicial $^{(1)}$ & Final & & & \\
\hline Trifluralin GrDA (500 g/1) & 750 & $139 a^{(2)}$ & $154 a$ & $1468 \mathrm{a}$ & $82,9 a$ & 13,9 \\
\hline Trifluralin GrDA $(500 \mathrm{~g} / 1)$ & 1000 & $161 \mathrm{a}$ & $159 a$ & $1678 \mathrm{a}$ & $86,2 \mathrm{a}$ & 12,6 \\
\hline Trifluralin CE $(600 \mathrm{~g} / 1)$ & 720 & $168 \mathrm{a}$ & $157 \mathrm{a}$ & $1715 \mathrm{a}$ & $84,2 a$ & 13,8 \\
\hline Trifluralin CE (445 g/l) & 667 & $143 a$ & $156 a$ & $1794 \mathrm{a}$ & $85,8 \mathrm{a}$ & 14,0 \\
\hline Testemunha capinada & --- & $169 \mathrm{a}$ & $168 \mathrm{a}$ & $1492 \mathrm{a}$ & $76,0 \mathrm{a}$ & 12,5 \\
\hline Testemunha sem capina & --- & $20 b$ & $74 b$ & $772 \mathrm{~b}$ & $76,9 a$ & 12,6 \\
\hline Média Geral & & 142 & 147 & 1480 & 82,0 & 13,3 \\
\hline Valor de F & & $13,9 * *$ & $4,5 * *$ & $33,9 * *$ & $3,1 *$ & $1,0 * *$ \\
\hline DMS & & 44,3 & 76,2 & 460 & 11,6 & -- \\
\hline C.V. $(\%)$ & & 13,5 & 23,1 & 14,3 & 6,2 & 11,3 \\
\hline
\end{tabular}

(1) Contagem realizada aos 15 dias após a aplicação

(2) As médias na mesma coluna, seguidas pela mesma letra, não diferem significativamente, pelo teste de Tukey a $5 \%$. 
Tabela 4. Efeito de formulações de trifulralin e doses no peso de sementes, índice de colheita mecânica e fitotoxicidade na soja. Uberaba, MG. 1994/95.

\begin{tabular}{|c|c|c|c|c|c|}
\hline \multirow[t]{2}{*}{ Tratamento } & \multirow{2}{*}{$\begin{array}{l}\text { Dose } \\
\text { (g/ha) }\end{array}$} & \multirow{2}{*}{$\begin{array}{l}\text { Peso de } 100 \\
\text { sementes } \\
\text { (g) }\end{array}$} & \multirow{2}{*}{$\begin{array}{l}\text { Colheita } \\
\text { mecânica } \\
\text { (1 a 5) }\end{array}$} & \multicolumn{2}{|c|}{$\begin{array}{l}\text { Fitotoxicidade } \\
\text { (EWRC } 1 \text { a 9) }\end{array}$} \\
\hline & & & & 7 DAA & $21 \mathrm{DAA}$ \\
\hline Trifluralin GrDA (500 g/l) & 750 & 14,8 & $3,5 b^{(1)}$ & 1,0 & 1,0 \\
\hline Trifluralin GrDA $(500 \mathrm{~g} / \mathrm{l})$ & 1000 & 15,4 & $3,9 \mathrm{~b}$ & 1,0 & 1,0 \\
\hline Trifluralin CE $(600 \mathrm{~g} / \mathrm{l})$ & 720 & 15,4 & $3,9 \mathrm{~b}$ & 1,0 & 1,0 \\
\hline Trifluralin CE $(445 \mathrm{~g} / \mathrm{l})$ & 667 & 15,6 & $4,0 \mathrm{~b}$ & 1,0 & 1,0 \\
\hline Testemunha capinada & --- & 15,3 & $5,0 \mathrm{a}$ & 1,0 & 1,0 \\
\hline Testemunha sem capina & --- & 15,1 & $1,0 \mathrm{c}$ & 1,0 & 1,0 \\
\hline Média Geral & & 15,3 & 3,6 & 1,0 & 1,0 \\
\hline Valor de F & & 0,4 n.s. & $108,4 * *$ & -- & -- \\
\hline DMS & & -- & 0,6 & -- & -- \\
\hline C.V. $(\%)$ & & 6,2 & 7,4 & & \\
\hline
\end{tabular}

(1) As médias na mesma coluna, seguidas pela mesma letra, não diferem significativamente entre si, pelo teste de Tukey a 5\%. DAA= dias após aplicação

Tabela 5. Efeito de formulações e doses de trifluralin no controle de Brachiaria decumbens e Brachiaria plantaginea, aos 30 e 45 dias após aplicação. Uberaba, MG. 1994/95.

\begin{tabular}{|c|c|c|c|c|c|}
\hline \multirow{2}{*}{ Tratamento } & \multirow{2}{*}{$\begin{array}{c}\text { Dose } \\
\text { (g/ha) }\end{array}$} & \multicolumn{2}{|c|}{ Brachiaria decumbens ${ }^{(1)}$} & \multicolumn{2}{|c|}{ Brachiaria plantaginea ${ }^{(1)}$} \\
\hline & & 30 DAA & 30 DAA & 45 DAA & 45 DAA \\
\hline Trifluralin GrDA $(500 \mathrm{~g} / \mathrm{l})$ & 750 & $84 c^{(2)}$ & $81 b^{(2)}$ & $85 \mathrm{c}$ & $89 \mathrm{~b}$ \\
\hline Trifluralin GrDA (500g/l) & 1000 & $87 \mathrm{c}$ & $87 \mathrm{~b}$ & $89 \mathrm{bc}$ & $91 \mathrm{~b}$ \\
\hline Trifluralin CE $(600 \mathrm{~g} / 1)$ & 720 & $90 \mathrm{bc}$ & $87 \mathrm{~b}$ & $92 \mathrm{~b}$ & $92 \mathrm{~b}$ \\
\hline Trifluralin CE (445 g/1) & 667 & $95 \mathrm{bc}$ & $89 \mathrm{~b}$ & $94 \mathrm{~b}$ & $97 \mathrm{~b}$ \\
\hline Testemunha capinada & --- & $100 \mathrm{a}$ & $100 \mathrm{a}$ & $100 \mathrm{a}$ & $100 \mathrm{a}$ \\
\hline Testemunha sem capina & --- & $0 \mathrm{~d}$ & $0 \mathrm{c}$ & $0 \mathrm{~d}$ & $0 \mathrm{c}$ \\
\hline Número de perfilhos $/ \mathrm{m}^{2}$ & & 52,0 & 30,0 & 27,0 & 39,5 \\
\hline Valor de F & & $327,1^{* *}$ & $461,9 * *$ & $518,5 * *$ & $285,0^{* *}$ \\
\hline C.V $(\%)$ & & 5,6 & 4,8 & 4,4 & 5,9 \\
\hline
\end{tabular}

DAA = dias após a aplicação

(1) Dados transformados em Arc. Seno $\sqrt{\mathrm{x} / 100}$ para a análise da variância.

(2) As médias na mesma coluna, seguidas pela mesma letra, não diferem significativamente, pelo teste de Tukey a $5 \%$.

\section{CONCLUSÕES}

As formulações GrDA e CE de trifluralin são eficientes no controle de Brachiaria decumbens e B. plantaginea na soja, sendo a segunda levemente superior no controle inicial de Brachiaria decumbens (15 dias após a aplicação) e final de B. plantaginea (45 dias após a aplicação).

\section{LITERATURA CITADA}

DARIO, G. J. A.; DARIO, P. N.; BALTIERI, E. M. Avaliação da eficiência do herbicida trifluralin no controle de plantas daninhas ocorrentes na cultura da soja (Glycine max) In: CONGRESSO BRASILEIRO DE HERBICIDAS E PLANTAS DANINHAS. 19; 1993 Londrina. Resumos... Londrina: SBHED, 1993, p. 126127.

LORENZI, H. J.; DAVIS, G. G. Competição de herbicidas na cultura da soja. In: SEMINÁRIO BRASILEIRO DE HERBICIDAS E ERVAS DANINHAS, 11; 1976, Londrina. Resumos...Londrina: SBHED/IAPAR/IBC-MA., 1976, p. 67-68.

MACHADO NETO, J. G.; BACILI, J. C.; TOLEDO, A. R. M.; FORLAN JUNIOR, E.; FUSTAINE, F.G. Seletividade de herbicidas aplicados em pré plan- 
Eficácia agronômica de formulações e doses...

tio incorporado, à cultura da soja (Glycine max) e eficiência no controle das plantas daninhas. In: CONGRES SO BRASILEIRO DE HERBICIDAS E PLANTAS DANINHAS, 17; 1988, Piracicaba. Resumos... Ribei rão Preto: Legis Summa, 1988, p. 110-112.

MAIA, A. C. Controle de plantas daninhas. Inf. Agropec., Belo Horizonte, v. 4, n. 43, 1978. p. 370-380.

MAIA, A. C.; LACA-BUENDIA, J. P del C.; REZENDE, A. M. Avaliação de mistura de herbicidas na cultura de soja (Glycíne max (L.) Merrill), cultivar UFV-1, em condições de cerrado. In: SEMINÁRIO NACIONAL DE SOJA, 2; 1991, Brasília. Anais... Londrina: EMBRAPA-CNPSO, 1991, p. 290-298. (EMPRAPACNPSo). (Documentos, I.).

MELHORANÇA, A. L.; VICTÓRIA FILHO, R. Efeito de profundidade de incorporação no solo de herbicidas residuais na cultura de soja (Glycíne $\max$ (L.) Merríl.) ln: CONGRESSO BRASILEIRO DE HERBICIDAS E ERVAS DANINHAS, 14; CONGRESO DE LA ASOCIACIÓN LATINOAMERICANA DE MALEZAS, 6; 1982, Campinas. Resumos... Campinas: SBHED/ALAM, 1982, p.80.

RAFAEL, J. 0. V.; MAIA, A. C.; SOUZA, I. F. de. Avaliação de herbicidas na cultura da soja (Glycíne max (L.) Merrill.), variedade Santa Rosa, no município de Uberaba-Minas Gerais, Brasil. In: Soja: Resumos Informativos, por Aloizio de Arruda Pinto et al. Brasília, 1978. v. 2. Ervas Daninhas e seu controle. p. 201-202.

RODRIGUES, J. J. V.; COELHO, J. P. Eficiência do imazaquim, isolado e em mistura com outros herbicidas, aplicados em pré-plantio incorporado, para o controle de plantas daninhas na cultura da soja (Glycine max). In: CONGRESSO BRASILEIRO DE HERBICIDAS E PLANTAS DANINHAS, 17; 1988. Piracicaba. Resumos...Ribeirão Preto: Legis Summa, 1988, p. 101 102.

SCALEA, M. J. Controle de plantas daninhas da soja (Gíycine $\max ($ L.) Merr.) em solos de cerrado. In: CONGRESSO BRASILEIRO DE HERBICIDAS E PLANTAS DANINHAS, 16; 1986, Campo Grande. Resumos... Campo Grande: SBHED, 1986, p. 65-66.

SEDIYAMA, T.; REIS, M. S. Cultura da soja em Minas Gerais (Instruções Práticas). Boletim de Extensão. UFV: Viçosa, 1990. 16p.

SILVA, M. J.; KISHINO, J. I. Estudo da dose de pendimethalin em função do método de aplicação na cultura de soja (Giycine max (L.) Merríll). In: CONGRESSO BRASILEIRO DE HERBICIDAS E PLANTAS DANINHAS, 16; CONGRESO DE LA ASOCIACIÓN LATINOAMERICANA DE MALEZAS, 7; 1984. Belo Horizonte. Resumos... Piracicaba:Augregaf, 1984. p. 88-89.

VICTORIA FILHO, R.; GARCIA, I.; CRUZ, L. S. P. Controle de plantas daninhas na cultura da soja (Glycine $\max ($ L.) Merril.) com herbicidas em pré-plantio incorporado. In: SEMINÁRIO BRASILEIRO DE HERBICIDAS E ERVAS DANINHAS, 11; 1976, Londrina. Resumos... Londrina: SBHED/IAPAR/IBC-MA., 1976. p. 73. 
Revista Brasileira de Herbicidas, v.2, n.1, 2001. 